Nervenarzt 2017 $\cdot 88: 1-2$

DOI 10.1007/s00115-016-0257-z

Online publiziert: 19. Dezember 2016

(c) Springer Medizin Verlag Berlin 2016

CrossMark

\section{A. Heinz ${ }^{1}$ F. Schneider ${ }^{2}$}

${ }^{1}$ Klinik für Psychiatrie und Psychotherapie, Campus Mitte, Charité - Universitätsmedizin Berlin, Berlin, Deutschland

${ }^{2}$ Klinik für Psychiatrie, Psychotherapie und Psychosomatik, Uniklinik RWTH Aachen, Aachen, Deutschland

\title{
Herausforderungen und Perspektiven
}

In den letzten zwei Jahren kam es zu einem deutlich verstärkten Zuzug von geflüchteten Personen und Migranten nach Deutschland, wobei die größte Zahl der betroffenen Personen aus den Kriegsgebieten Syriens, Afghanistans und des Iraks stammten [1].

Innerhalb Europas kam die größte Zahl geflüchteter Menschen nach Deutschland, wobei direkt an die Kriegsgebiete angrenzende Länder, wie die Türkei und der Libanon, noch wesentlich mehr Personen aufnahmen [2]. Menschen mit Migrationshintergrund und geflüchtete Personen haben psychische Erkrankungen nicht nur in vergleichbarem Ausmaß wie die einheimische Bevölkerung, sie haben zudem besondere Risiken bezüglich des Auftretens traumabedingter psychischer Störungen [3-6].

\section{) Sprach- und Kulturmittler müssen verstärkt in Anspruch genommen werden}

Die akute und längerfristige Versorgung dieser Personengruppe stellt besondere Anforderungen an unser Gesundheitssystem, da hier die bisher von den Krankenkassen nicht finanzierten Sprachund Kulturmittler mit ihren Dolmetscherleistungen verstärkt in Anspruch genommen und damit finanziert werden müssen [7]. Dies gilt gerade bei psychischen Erkrankungen mit komplexen Entstehungsbedingungen, in denen Familienmitglieder oder sprachkundige, aber nicht in der Übersetzertätigkeit geschulte Personen oft nicht hilfreich und ggf. sogar schädlich wirken können, z. B. wenn es um persönliche Verletzungen oder Traumatisierungen geht.

Das vorliegende Schwerpunktheft widmet sich der Frage, wie die Gesundheitsversorgung dieser Personen im Bereich der Psychiatrie, Psychotherapie und Psychosomatik gestaltet werden sollte. Sukale und Kollegen gehen hier auf die besonderen Bedürfnisse minderjähriger Flüchtlinge ein, die einfach anzuwendende Instrumente erfordern, um Belastungsfaktoren wie Ressourcen angemessen $\mathrm{zu}$ erfassen und in die Hilfeplanung einzubeziehen. Die Autoren stellen entsprechende Untersuchungsinstrumente vor, die im Rahmen praktischer klinischer Tätigkeit erprobt wurden und gut anwendbar sind.

Schneider, Bajbouj und Heinz beschreiben ein Modell eines gestuften Vorgehens für die psychische Versorgung von Flüchtlingen in Deutschland, welches auf niedrigschwellige Angebote im Sinne von Peer-to-Peer-Beratern und internetbasierten Interventionen beruht und darauf abgestuft aufbauend expertengeleitete Gruppeninterventionen und schließlich Einzeltherapien vorsieht. Besonderes Gewicht kommt hierbei der Aktivierung der Ressourcen der geflüch- teten Menschen zu, die z. B. im Rahmen der Peer-to-Peer-Beratung aktiv werden können.

Maercker und Mitarbeiter fokussieren in ihrem Beitrag auf das Konzept der sozialen Unterstützung, welche für traumatisierte Menschen besondere Bedeutung hat. Die Autoren erläutern wichtige Ergebnisse der Forschung zur sozialen Unterstützung hinsichtlich ihrer klinischen Bedeutung und differenzieren zwei traumaspezifische Ansätze dieses Konzeptes, einerseits die Fokussierung auf Wertschätzung als Überlebende eines Traumas und andererseits die Offenlegung der traumatischen Erfahrungen.

Schließlich erläutern Elbert und Kollegen Erfahrungen aus der Arbeit mit traumatisierten Geflüchteten in Krisen- und Kriegsregionen und ihre Übertragbarkeit auf die Situation in Deutschland. Sie beschreiben dabei Behandlungsverfahren, deren Wirksamkeit durch wissenschaftliche Studien belegt sind, und erläutern ein Kaskadenmodell, in dem Mitarbeiter der jeweiligen Flüchtlings- und Exilgemeinschaften Personen identifizieren können, die eine ausführliche Diagnostik und Therapie benötigen. Auch hier liegt der Schwerpunkt auf der Ausbildung zweisprachiger Personen, die im Rahmen ihrer Berufsausbildung z. B. als Lehrerinnen oder Pflegemitarbeiter weitergebildet werden können, um unter Su- 
pervision Diagnostik und Interventionsmodule anzuwenden.

Schließlich erläutert in einem Interview Frau Missmahl, die langjährig in Afghanistan psychosoziale Berater ausgebildet hat, ihren personenzentrierten Ansatz im Gespräch mit Martin Bohus [8].

\section{》) Bestehende Ressourcen müssen unterstützt und gefördert werden}

Den hier vorgestellten Ansätzen gemeinsam ist der Fokus auf die psychosoziale Unterstützung der geflüchteten Personen im Sinne gestufter Interventionen, bei denen ein starkes Gewicht auf die Unterstützung und Förderung bestehender Ressourcen, die Fortbildung der Geflüchteten und ihr „empowerment “ zur gegenseitigen Hilfe liegt. Im Sinne salutogenetischer Ansätze [9] soll dies bei den weitergebildeten Personen selbst, aber auch in ihrem Umfeld die Fähigkeiten und Fertigkeiten im Umgang mit den Herausforderungen der neuen Lebenssituation stärken und helfen, Verständigungsschwierigkeiten zu verringern, den $\mathrm{Zu}$ gang zum Gesundheitssystem zu verbessern und eine an den Bedürfnissen der betroffenen Personen orientierte Versorgung sicherzustellen. Ungeachtet dieser Modelle müssen die Behandlerinnen und Behandler sich kulturell und methodisch, z. B. im Hinblick auf Hinzuziehung von Dolmetschern, auf diese neue Patientengruppe einstellen.

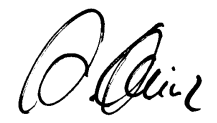

Prof. Dr. Dr. phil. Andreas Heinz

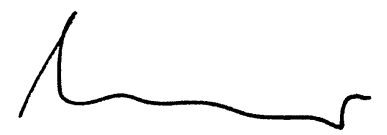

Prof. Dr. Dr. rer. soc. Frank Schneider

\section{Korrespondenzadresse}

\section{Prof. Dr. Dr. phil. A. Heinz}

Klinik für Psychiatrie und Psychotherapie, Campus Mitte, Charité - Universitätsmedizin Berlin

Charitéplatz 1, 10117 Berlin, Deutschland andreas.heinz@charite.de

Interessenkonflikt. A. Heinz und F. Schneider geben an, dass kein Interessenkonflikt besteht.

\section{Literatur}

1. BAMF (2016) Asylgeschäftsstatistik für den Monat Juni 2016. https://www.bamf.de/SharedDocs/ Anlagen/DE/Downloads/Infothek/Statistik/Asyl/ 201606-statistik-anlage-asyl-geschaeftsbericht. pdf? blob=publicationFile. Zugegriffen: 02. Nov 2016

2. Mediendienst Integration (2016) https:// mediendienst-integration.de/migration/fluchtasyl/zahl-der-fluechtlinge.html. Zugegriffen: 02 . Nov 2016

3. Crumlish N, O'Rourke K (2010) A systematic review of treatments for post-traumatic stress disorder among refugees and asylum-seekers. J Nerv Ment Dis 198(4):237-251

4. Glaesmer H, Brähler E, Wittig U, Martin A, Mewes R, Rief W (2009) Sind Migranten häufiger von psychischen Störungen betroffen? Eine Untersuchung an einer repräsentativen Stichprobe der deutschen Allgemeinbevölkerung. Psychiatr Prax 36:16-22

5. Schouler-Ocak M, Aichberger MC, Penka S, Kluge U, Heinz A (2015) Psychische Störungen bei Menschen mit Migrationshintergrund in Deutschland. Bundesgesundheitsblatt 58(6):527-532

6. Swinnen SG, Selten JP (2007) Mood disorders and migration:meta-analysis. Br J Psychiatry 190:6-10

7. Kluge U, Bogic M, Devillé W, Greacen T, Dauvrin M, Dias S, Gaddini A, Jensen NK, loannidi- Kapolou E, Mertaniemi R, Puipcinós i Riera R, Sandhu $S$, Sarvary A, Soares JJF, Stankunas M, Straßmayr C, Welbel M, Heinz A, Priebe S (2012) Health services and the treatment of immigrants: data on service use, interpreting services and immigrant staff members in services across Europe. Eur Psychiatry 27:S56-S62

8. Missmahl I, Kluge U, Bromand Z, Heinz A (2012) Teaching psychiatry and establishing psychosocial services-lessons from Afghanistan. Eur Psychiatry 27:S76-S80

9. Antonovsky A (1997) Salutogenese: Zur Entmystifizierung der Gesundheit. Forum für Verhaltenstherapie und Psychosoziale Praxis, Bd. 36. dgvt, Tübingen
Auch online Zugang zu allen Beiträgen Ihres Individualabonnements

Wussten Sie, dass Sie als Abonnent dieser Zeitschrift automatisch online Zugriffsrechte auf das gesamte Beitragsarchiv und die CME-Kurse haben?

Der Zugang zu Ihrer Online-Bibliothek und den CME-Kursen ist im Abonnement Ihrer Zeitschrift inbegriffen. Neben den gedruckten Ausgaben, können Sie Themen einfach am Computer recherchieren. Für den Zugang müssen Sie sich lediglich einmal über www.springermedizin.de/register registrieren.

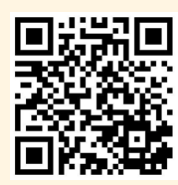

Über diesen QR-Code schnell und einfach registrieren

Für die Registrierung brauchen Sie nur

- die Nummer Ihres Abonnements

(siehe Adressaufkleber auf Ihrem Heft) und - Ihre Lieferanschrift.

So stellen Sie die Zugehörigkeit zu Ihrer Zeitschrift sicher.

Nach Login erhalten Sie dann sofort die Online-Rechte für den Volltextzugriff und den Zugang zu Ihren CME-Kursen.

Mit Benutzername und Passwort haben Sie außerdem Zugang zu den freien Inhalten auf den Seiten von:

https://www.springermedizin.de/ http://www.aerztezeitung.de/ http://www.heilberufe-online.de/

Sollten Fragen oder Probleme auftauchen, wenden Sie sich einfach an Ihren Kundenservice über:

kundenservice@springermedizin.de 